ORIGINAL ARTICLE

\title{
Is virtual bronchoscopy useful for physicians practising in a district general hospital?
}

\author{
K Dheda, C M Roberts, M R Partridge, I Mootoosamy
}

Postgrad Med J 2004;80:420-423. doi: 10.1136/pgmi.2003.013946

See end of article for authors' affiliations .....................

Correspondence to: Dr Keertan Dheda, Chest Unit, Whipps Cross University Hospital NHS Trust, Leytonstone, London EII INR, UK; k.dheda@ud.ac.uk

Submitted 26 August 2003 Accepted 4 October 2003
Background: Virtual bronchoscopy software is now available to district general hospitals (DGHs). There is limited information on the clinical utility of virtual bronchoscopy and whether it offers any additional information over conventional axial computed tomography in the setting of a busy DGH chest unit. Methods: Virtual bronchoscopy and computed tomography findings were compared in all patients who had a virtual bronchoscopy study over a 12 month period.

Results: Eighteen consecutive patients had virtual bronchoscopy for a specific clinical indication over the study period. Additional information was conveyed by virtual bronchoscopy in five patients (in four patients the airways distal to an obstruction were better visualised thereby influencing decisions about airway stenting and in one patient the virtual bronchoscopy study showed an endobronchial lesion missed on computed tomography). In nine patients who were unfit for fibreoptic bronchoscopy (FOB) the radiologist was more confident in excluding an obstructive airway lesion. The main indication for performing a virtual bronchoscopy study was to rule out an obstructive airway lesion in patients who were unfit for FOB $(n=11)$.

Conclusion: Virtual bronchoscopy is feasible and useful in the management of a few selected patients in a DGH chest unit. Virtual bronchoscopy may convey additional information over computed tomography when the distal airways need to be visualised and for discrete endoluminal lesions.
$\mathrm{T}$ he availability of fibreoptic bronchoscopy (FOB) and computed tomography has revolutionised the diagnostic work-up of chest diseases. Although FOB enables direct visualisation of the bronchial mucosa, the operator is unable to see beyond an obstructive lesion and not all patients may tolerate the procedure. The limitations of conventional axial tomographic scans include limited accuracy in the assessment of bronchial stenosis and of airways that course oblique to the axial plane. ${ }^{1}$

More recently spiral computed tomographic scanners have become widely available to district general hospitals (DGHs). In addition to these scanners allowing faster imaging times, lower radiation doses and fewer motion artefacts, they also acquire continuous data between the actual slices displayed. ${ }^{2}$ By using available computer software this complete data can be retrieved and reprocessed to create a three dimensional virtual bronchoscopy of the tracheobronchial tree. ${ }^{34}$ These virtual views can be displayed as both three dimensional reconstructions and as two dimensional images in the sagittal or coronal plane (multiplanar reconstructions) (fig 1). With multiplanar reconstructions the contents of the airway and the extraluminal hilar and mediastinal structures may be viewed simultaneously. The viewer is also able to rotate images and repeatedly navigate through the inside of the tracheobronchial tree as if in a simulated real life bronchoscopy.

Various studies have shown a correlation between virtual bronchoscopy and FOB findings in patients known to have airway stenosis or bronchial obstruction. ${ }^{5-11}$ However there are few data on what additional information virtual bronchoscopy provides over computed tomography in a clinical setting. ${ }^{12}$ This is important as virtual bronchoscopy is within reach of many DGHs that have spiral computed tomography scanners and the software to perform multiplanar reconstruction and virtual bronchoscopy. We reviewed our experience of virtual bronchoscopy to determine if this new technology added any value to the respiratory assessment of patients in a DGH.

\section{PATIENTS AND METHODS}

We reviewed the virtual bronchoscopy, computed tomography, and FOB findings in 18 consecutive patients (12 males and six females, age range $25-88$ years), who had a virtual bronchoscopy study performed over a 12 month period. Virtual bronchoscopy studies were requested at the discretion of chest physicians whenever they thought that it might be helpful for patient management. Subjects referred to as "unfit for FOB" were those with hypoxia (oxygen tension of between 5.5 to $7.8 \mathrm{kPa}$ in room air) or low blood pressure (systolic blood pressure $\leqslant 90 \mathrm{~mm} \mathrm{Hg}$ ).

Computed tomography images were acquired using an IGE HiSpeed LX/I scanner ( $120 \mathrm{kV}, 200-220 \mathrm{~mA}, 5 \mathrm{~mm}$ collimation, reconstruction interval of $2.5 \mathrm{~mm}$, pitch of 1.5-2.0, and after $100 \mathrm{ml}$ intravenous contrast). The acquisition time was 20-22 seconds. To obtain good quality virtual bronchoscopy and multiplanar reconstructions a limited area of the tracheobronchial tree was also scanned to cover the trachea, carina, and main bronchi. This was done at a finer collimation and higher pitch, thereby giving a shorter acquisition time. For endobronchial views the scanned images were then transferred to a GE Advantage Windows Workstation (Navigator, GE Medical Systems, Milwaukee, WI). This view also indicates the tip of the "endoscope" (fig 1), which could be rotated in any direction as it was advanced down the bronchial tree. Two radiologists reported the images without any prior knowledge of the bronchoscopic findings of the patient. The chest physician, however, had access to the virtual bronchoscopy findings if the patient later underwent FOB. Ethical approval was obtained from the Waltham Forest and Redbridge Ethics Committee.

Data were recorded regarding indication for virtual bronchoscopy, timing relative to FOB, and information acquired from virtual bronchoscopy compared with computed

Abbreviations: $D G H$, district general hospital; FOB, fibreoptic bronchoscopy 

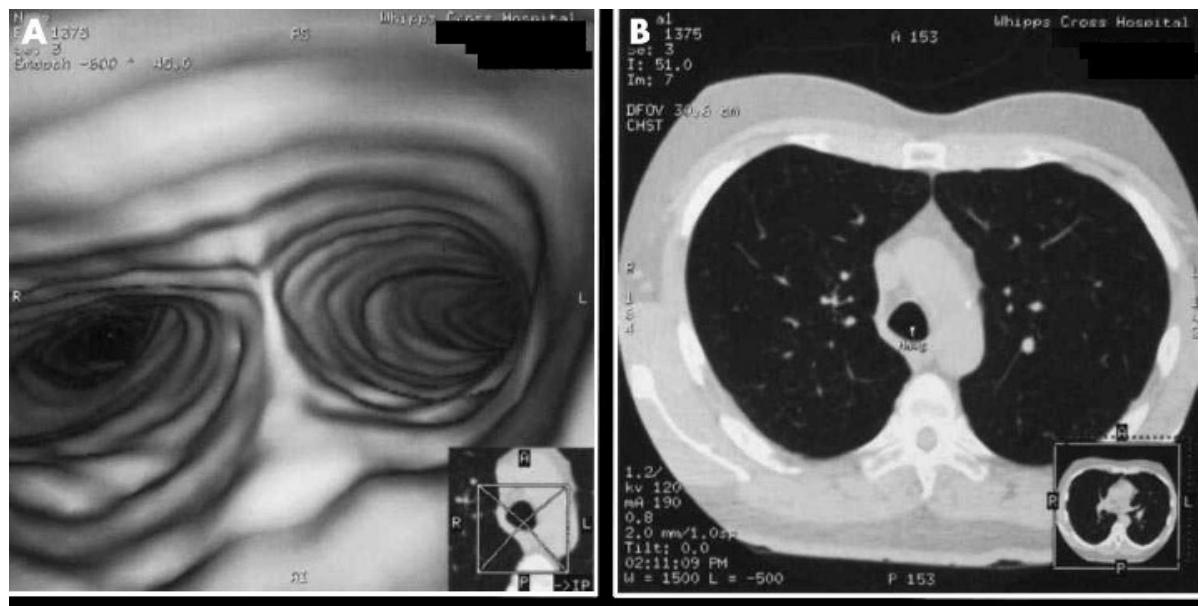

Figure 1 Multiview mode showing the virtual bronchoscopy image of a normal carina (A), axial computed tomography image (B) and multiplanar reconstructions (C and D). The white navigation beacon in the sagittal and coronal planes indicates the tip of the "virtual bronchoscope", which can be rotated in any direction to navigate down a desired airway.
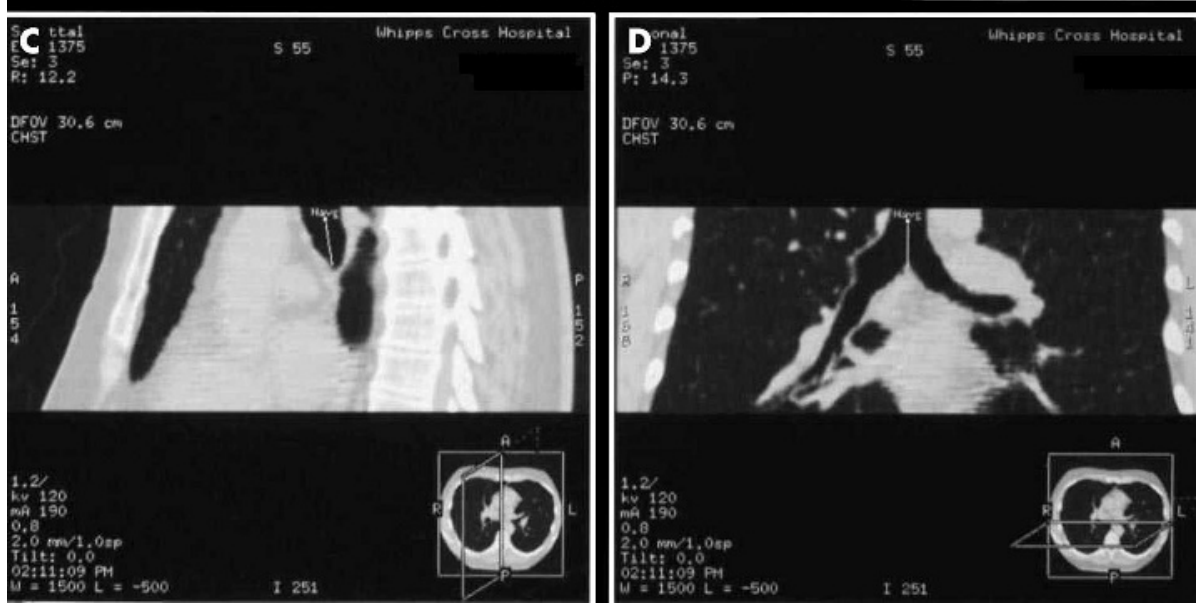

tomography with respect to (1) the patency of distal airways, (2) endobronchial lesions seen on virtual bronchoscopy but not on axial computed tomography, and (3) whether a central obstructive lesion could be more confidently excluded. Where available, the FOB findings and concordance with virtual bronchoscopy were noted.

\section{RESULTS}

Eighteen consecutive patients underwent virtual bronchoscopy over a 12 month period. In comparison 321 FOBs were performed in our hospital during the same period. The virtual bronchoscopy study was adequate in all patients but one patient had some breathing artefact. Virtual bronchoscopy was performed before FOB in patients unfit for the procedure; the time between virtual bronchoscopy and FOB in these patients was between one and 20 days except in one patient where it was eight weeks. Patients who underwent FOB before virtual bronchoscopy were mainly those who had a diagnosis of lung cancer that had been made bronchoscopically some months before. In these cases virtual bronchoscopy was used to determine whether obstructive lesions had become more advanced or to assess the patency of distal airways. On this basis, virtual bronchoscopy was performed before FOB in five patients, after FOB in seven patients (one patient had a rigid bronchoscopy and one patient had fibreoptic laryngoscopy) and FOB was not done in six patients.

Additional information was conveyed by virtual bronchoscopy in five patients (in four patients the airways distal to an obstruction were better visualised thereby influencing deci- sions about airway stenting, and in one patient the virtual bronchoscopy study showed an endobronchial lesion missed on computed tomography). In nine patients with or without known malignancy who were unfit for FOB the radiologist was more confident in excluding an obstructive airway lesion. In three of these patients the normal virtual bronchoscopy findings obviated later FOB.

In the 12 patients who had FOB and virtual bronchoscopy, the findings concurred in all patients (both studies were normal in five patients and virtual bronchoscopy detected stenosis in all seven patients where it was demonstrated on FOB). In one patient however, although virtual bronchoscopy showed the left main bronchial stenosis, it did not demonstrate $2 \mathrm{~mm}$ mucosal nodules in the right main bronchus. The main advantage of $\mathrm{FOB}$ was the ability to make a histological diagnosis in five patients.

The three main indications for performing a virtual bronchoscopy study were: (1) patients unfit for FOB where an obstructive airway lesion needed to be excluded $(\mathrm{n}=7)$, (2) to rule out an obstructive lesion in patients with known lung cancer who were unfit for $\operatorname{FOB}(n=4)$, and (3) for evaluation of airways patency distal to an endobronchial obstruction $(n=2)$. Other situations in which virtual bronchoscopy was found to be useful in our study included evaluation of the larynx after trauma, evaluation of a smoker with haemoptysis and warfarin toxicity, airway evaluation in cavitatory disease, and in patients with poorly tolerated FOB where certain parts of the central airways were not adequately visualised. Described below, are some brief case histories illustrating the usefulness of virtual bronchoscopy in specific clinical situations. 


\section{(1) Exclusion of airway obstruction in patients unfit for FOB}

Patient A (an 82 year old man) had a right upper lobe pneumonia on computed tomography and was unfit for FOB. Virtual bronchoscopy showed a polypoid lesion in the right upper lobe bronchus (fig 2A and 2B), which was not initially seen on axial computed tomography (fig 2C). Later FOB confirmed a carcinoid tumour (fig 3).

Patient B had a previous tracheal polypectomy and presented with acute onset of dyspnoea which would have necessitated an urgent rigid bronchoscopy. The three dimensional virtual bronchoscopy confidently excluded an endoluminal lesion. Later FOB was normal.

\section{(2) Patients with known lung cancer unfit for FOB}

Patient $\mathrm{C}$ who was diagnosed eight months previously with lung cancer, presented to hospital with dyspnoea and was unfit for FOB. Virtual bronchoscopy clearly delineated an airway obstruction and a multiplanar reconstruction confirmed a mainly endobronchial tumour. The patient was referred for laser resection.

\section{(3) Patency of distal airways}

Patient D was investigated for left lower lobe collapse. FOB showed a slit-like left mainstem bronchus and histological examination of bronchial mucosa revealed a squamous carcinoma. A virtual bronchoscopy study was undertaken to evaluate airways distal to the obstructive lesion to assist with decision making regarding tertiary referral. While computed tomography showed a mass compressing the left main bronchus, virtual bronchoscopy clearly showed patent distal airways and a stent was inserted.

\section{DISCUSSION}

A review of our experience with virtual bronchoscopy in a busy DGH chest unit indicates that it is useful for the clinical management of selected patients by providing additional information when compared with computed tomography. We also found a good correlation between virtual bronchoscopy and FOB findings as others have previously reported. . $^{5-11}$ However these studies focused on determining the sensitivity and concordance of virtual bronchoscopy with respect to a specific bronchoscopic diagnosis. Instead our study examined how virtual bronchoscopy was actually being used in a clinical context. The main advantage of virtual bronchoscopy in a clinical setting was to provide a diagnostic tool for airway evaluation in patients who were not suitable for FOB on presentation to hospital. Unlike previous studies, we found virtual bronchoscopy particularly helpful in patients with

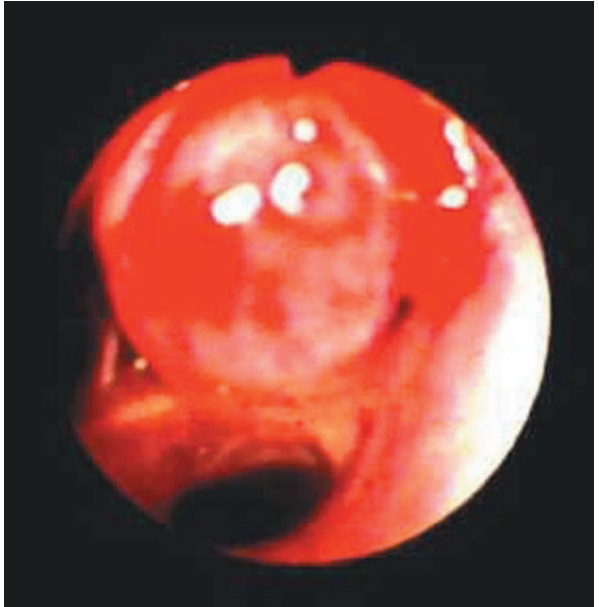

Figure 3 The later FOB appearance of a carcinoid tumour in the right upper lobe bronchus of patient $A$.

known lung cancer who were unfit for FOB. This group often poses a difficult management problem for clinicians and we found virtual bronchoscopy valuable to assess the presence and degree of airway obstruction. An additional advantage of virtual bronchoscopy reconstruction software over FOB in this context, is the ability to determine the length of and see beyond a stenosis. This may be helpful in decisions regarding palliative tertiary care referral for stenting or laser resection..$^{1013}$ We also found virtual bronchoscopy to be clinically useful in evaluating patients with suspected laryngotracheal pathology where FOB may be hazardous or initially unfeasible.

Our report highlights the major shortcomings of virtual bronchoscopy when used in a clinical setting. We have shown that virtual bronchoscopy may miss mucosal or infiltrative changes; this was previously reported by Fleiter and colleagues who found that high grade stenoses were easily visualised but discrete infiltrative changes were missed or underestimated in $25 \%$ of patients. ${ }^{8}$ The other limitation of virtual bronchoscopy is the inability to obtain a histological diagnosis. Therefore virtual bronchoscopy cannot replace FOB in a clinical setting and as such it is unlikely to shorten FOB waiting times in DGHs.

The suboptimal images obtained in one patient due to respiratory movements demonstrate that virtual bronchoscopy may cause false negative examinations because of breathing artefacts or mucus in the airways. ${ }^{4}$ Although many
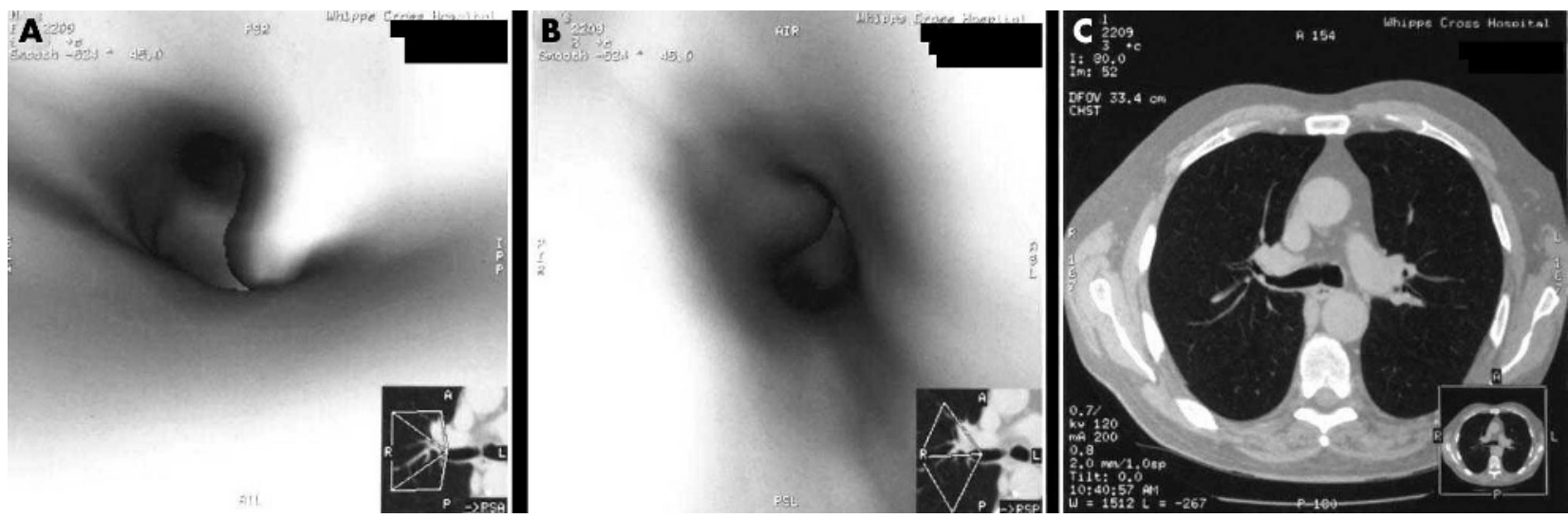

Figure 2 Patient $A$ was initially unfit for FOB. The virtual bronchoscopy shows a polypoid lesion in the right upper lobe bronchus (A and B), which was not obvious and initially missed on axial computed tomography cuts (C). 
of our patients were hypoxic, we were able to minimise the effects of breathing movements by keeping acquisition times to about $12-15$ seconds by increasing the pitch to $2-2.5$ and up to 3 in one or two patients. To get better image quality we also used finer collimation and reconstruction intervals over a limited area. We did, however, do an initial complete examination of the whole chest using $5 \mathrm{~mm}$ collimation.

Although the small number of patients and selection bias of the attending physicians are limitations of this study, we have shown that virtual bronchoscopy is feasible and useful for the imaging of selected patients in the setting of a DGH. Virtual bronchoscopy is particularly useful to study the central airways if FOB is not possible and as an aid to stenting as airways distal to an endobronchial obstruction may be better visualised. We would like to emphasise that virtual bronchoscopy has a limited role in a few highly selected patients for the indications described above. Whether this provides any tangible benefit for patients and its predictive value over computed tomography in a specific clinical context requires further study, and is the subject of a prospective ongoing trial at our institution.

\section{Authors' affiliations}

K Dheda, C M Roberts, M R Partridge, Chest Unit, Whipps Cross

University Hospital NHS Trust, London, UK

I Mootoosamy, Department of Radiology, Whipps Cross University Hospital NHS Trust, London, UK

\section{REFERENCES}

1 Nadich DP, Gruden JF, McGuinness G, et al. Volumetric (helical/spiral) CT (VCT) of the airways. J Thorac Imaging 1997; 12:11-28.

2 Kalender WA, Seissler W, Klotz E, et al. Spiral volumetric CT with singlebreath hold technique, continuous transport and continuous scanner rotation. Radiology 1990;176:181-3.

3 Higgins WE, Ramaswami K, Swift RD, et al. Virtual bronchoscopy for 3dimensional pulmonary image assessment: state of the art and future needs. Radiographics 1998;18:761-78.

4 Aquino SL, Vining DJ. Virtual bronchoscopy. Clin Chest Med 1999:20:725-30.

5 Ferretti GR, Knoplioch J, Bricault I, et al. Central airway stenoses: preliminary results of spiral-CT-generated virtual bronchoscopy simulations in 29 patients. Eur Radiol 1997;7:854-9.

6 Vining DJ, Liu K, Choplin RH, et al. Relationships of virtual reality endobronchial simulations to actual bronchoscopic findings. Chest 1996; 109:549-53.

7 Liewald F, Lang G, Fleiter T, et al. Comparison of virtual and fibreoptic bronchoscopy. Thorac Cardiovasc Surg 1998;46:361-4.

8 Fleiter T, Merkle EM, Aschoff AJ, et al. Comparison of real-time virtual and fibreoptic bronchoscopy in patients with bronchial carcinoma: opportunities and limitations. Am J Roentgenol 1997; 169:1591-5.

9 Cicero R, Criales J, Lorenzo JM. Flexible bronchoscopy and helical computed tomography with three dimensional reconstructions in a non-small cell carcinoma of the lung. J Bronchol 1998;5:98-103.

10 Kauczor HU, Wolcke B, Fischer B, et al. Three dimensional helical CT of the tracheobronchial tree: evaluation of imaging protocols and assessment of suspected stenoses with bronchoscopic correlation. Am J Roentgenol 1996;167:419-24.

11 Finkelstein SE, Summers RM, Nguyen DM, et al. Virtual bronchoscopy for evaluation of malignant tumours of the thorax. J Thorac Cardiovasc Surg 2002;123:967-72.

12 Haponik EF, Aquino SL, Vining DJ. Virtual bronchoscopy. Clin Chest Med 1999:20:201-17.

13 Lacrosse M, Trigaux JP, Van Beers BEWP. 3D spiral CT of the tracheobronchial tree. J Comput Assist Tomogr 1995; 19:341-7. 